\title{
Campañas de promoción sobre planificación familiar y su influencia en gestantes usuarias de la Red de Salud de San Juan de Miraflores, Lima- Perú
}

Sabrina Morales Alvarado l,a

\section{RESUMEN}

Con el objetivo de determinar el impacto de las campañas de promoción sobre planificación familiar (PF), en el nivel de conocimientos, actitudes y prácticas de las gestantes usuarias de la Red de salud San Juan de Miraflores (SJM), se realizó un estudio observacional, prospectivo, analítico de corte transversal desde el 15 de enero al 15 de Julio, 2013. Se encuestaron a 271 gestantes en cinco establecimientos de la jurisdicción. El 53.1\% habían nacido en Lima, predominando el rango de 19 a 35 años (83.7\%). El 74.2\% manifestó haber escuchado o visto alguna vez algún mensaje sobre PF en algún medio de comunicación. El $65.7 \%$ manifestó haberlos visto, pero solo $25.8 \%$ los leyó y comprendió.

Se concluye que los conocimientos sobre la PF en gestantes fueron limitados; y sus conocimientos, actitudes y prácticas sobre la planificación familiar, no tuvieron como fuente principal los medios de comunicación masiva.

(Horiz Med 2014; 14(2): 46-52)

Palabras clave: Campañas de promoción, planificación familiar, actitudes, conocimientos, prácticas. (Fuente: DeCS BIREME).

Impact of promotional campaigns on family planning and its influence on the knowledge, attitudes and practices of expectant users of the Red de Salud de San Juan de Miraflores, Lima-Perú

\begin{abstract}
In order to determine the impact of the campaigns of promotion on the family planning in relation to the knowledge, attitudes and practices in users pregnant of the network of health San Juan de Miraflores (SJM). An analytical, prospective, transversal, observational study was conducted from January 15 to July 15, 2013. 271 surveys were applied in women pregnant belonging to of five Health Centers of the jurisdiction. $53.1 \%$ of mothers pregnant were born in Lima. The predominant age was of the range 19 to 35 years (83.7\%). $74.2 \%$ demonstrated to have listened or saw at some time some message on the topic in some way of communication. $65.7 \%$ demonstrated to have seen them, but only $25.8 \%$ read and understood them.

It follows that knowledge on the family planning in mothers pregnant is limited and the answers of the mothers pregnant to the questionnaire like expression of her knowledge, attitudes and practices on the family planning, they did not take as a principal source of received information the media of massive communication. (Horiz Med 2014; 14(2): 46-52)
\end{abstract}

Key words: Promotion campaigns, family planning, attitudes, knowledge, practices. (Source: MeSH NLM).

\footnotetext{
${ }^{1}$ Consultora de proyectos de intervención en salud reproductiva. Lima, Perú.

a Doctora en ciencias de la Salud
} 


\section{INTRODUCCIÓN}

Un indicador significativo es la cobertura en planificación familiar, que influye directamente sobre la Salud materna y perinatal.

El Perú, aún afronta cifras altamente significativas de morbilidad y mortalidad materna, las cuales no se han logrado reducir significativamente, a pesar del impulso que se ha dado a programas masivos de atención y promoción de la salud en los medios de comunicación para incrementar la planificación familiar (1).

No se tienen datos acerca de la efectividad de la promoción de métodos anticonceptivos en medios y su impacto sobre los conocimientos, actitudes y prácticas de la población de madres gestantes en el Perú (2).

Tampoco se cuenta con suficiente información sobre los resultados que ha dado la promoción y difusión de estos servicios de atención de la mujer, lo cual constituye un vacío urgente de cubrir ya que como estrategia de gestión en búsqueda de una salud reproductiva, el desconocimiento, mal conocimiento o inadecuado mensaje en la promoción que se hace sobre la planificación familiar se traduce en la ausencia de adecuados conocimientos, actitudes y prácticas sobre el método más adecuado para espaciar un embarazo entre las madres gestantes de nuestro país.

El presente estudio tuvo como objetivo investigar el impacto de las campañas de promoción sobre la planificación familiar, realizada a través de diversos medios de comunicación y su influencia sobre los conocimientos, actitudes y prácticas de la población de gestantes usuarias de la Red de salud de San Juan de Miraflores.

\section{EL ESTUDIO}

Se realizó un estudio observacional, prospectivo, descriptivo y analítico a gestantes que acudían a su control prenatal a establecimientos pertenecientes a la Red de Salud SJM en Lima - Perú, entre el 15 de Enero y el 15 de Julio de 2013 y que voluntariamente aceptaron participar en el estudio.

El tamaño de la muestra se calculó teniendo en cuenta un nivel de confiabilidad del $95 \%$, la frecuencia de las variables de estudio como son varias se tomó la máxima frecuencia esperada que es el $50 \%$ y aceptando un error aleatorio de $5 \%$ en el estudio.

Se utilizó la selección de muestreo no probabilístico por muestreo consecutivo.

Se diseñó una encuesta conformada por 8 preguntas matrices, revisada y validada por juicio de expertos previa fase piloto, las que incluyeron datos generales y obstétricos de las gestantes encuestadas y preguntas sobre sus conocimientos, actitudes y prácticas relacionados a la planificación familiar y asociadas o no a las campañas de promoción sobre las mismas áreas de atención.

La recolección de datos se realizó mediante la aplicación de la encuesta validada, la cual tuvo un promedio de duración de 15 minutos aproximadamente.

Los datos se almacenaron en el programa Excel de Windows XP Profesional y se procesaron utilizando el programa estadístico SPSS 19.0

\section{HALLAZGOS}

Se aplicó 271 encuestas. En Centros de Salud: Manuel Barreto (62), Ollantay (54), Leonor Saavedra (64), Trébol Azul (33) y Pampas de San Juan (58).

El 53.1\% nació en Lima. Predominó el grupo de 19 a 35 años (83.7\%) y las convivientes (54.6\%). El $81.9 \%$ eran católicos, $6.3 \%$ evangélicos, $10 \%$ no se identificó con ninguna religión y $1.8 \%$ refirió otras religiones.

A la pregunta si habían escuchado alguna vez la frase "planificación familiar", el 74.2\% refirió haber escuchado. Figura 1. 


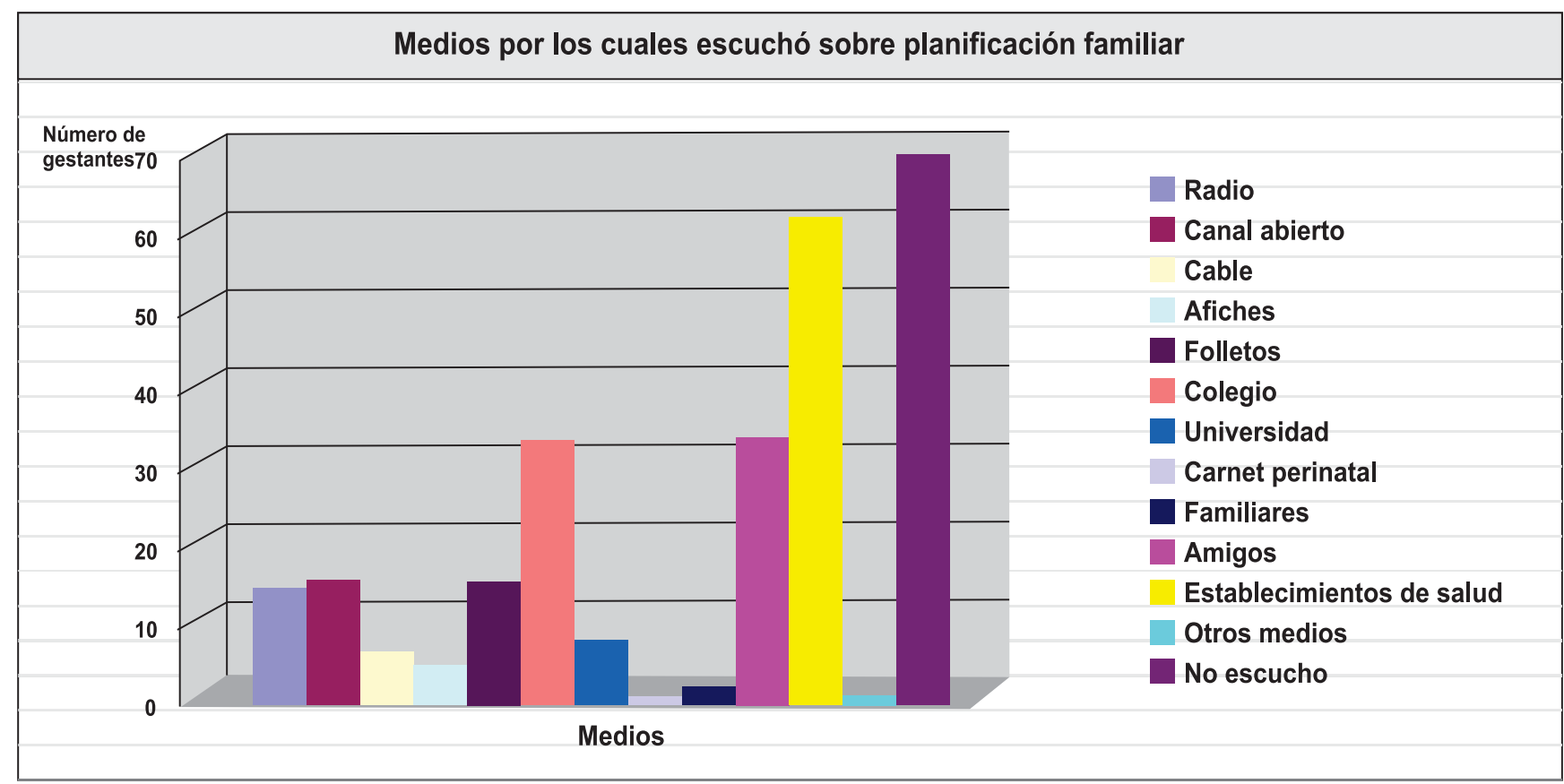

Figura 1. Medios por los cuales escuchó sobre planificación familiar

Con relación al mensaje que recuerdan al haber escuchado o visto sobre planificación familiar, el $38.4 \%$ refirió que se hablaba de saber como usar métodos anticonceptivos, el $21.8 \%$ refiere sobre cuántos hijos tener, $3.7 \%$ que se trata de cómo cuidarse para no contagiarse de ITS, 3.0\% refiere que es bueno para la salud de la mujer, $1.8 \%$ refiere que es para que la pareja viva saludablemente y el $4.8 \%$ no recuerda nada sobre el mensaje, mientras que el $25.8 \%$ no escuchó nunca nada sobre planificación familiar en ningún medio.

De las madres que escucharon mensajes sobre planificación familiar 63.8\% (173), lo consideraron importante.

Sobre si comprendieron o no el mensaje escuchado, $63.1 \%$ refirió haber comprendido y el $11.1 \%$ no comprendió nada.

Sobre si creían que es adecuado planificar la familia, el 88.2\% refirió que sí. Figura 2.

Del total de madres encuestadas, solo el $16.1 \%$ refirió haber planificado sus anteriores embarazos mientras que el $83.9 \%$ refirió no haberlo hecho.
Al preguntárseles sobre si habían pensado cuidarse con algún método anticonceptivo después del parto, $80.4 \%$ manifestó haberlo pensado, a través de: método hormonal inyectable $(19.5 \%)$, pastillas hormonales $(12.2 \%)$, DIU $(8.9 \%)$, preservativos (2.2\%) y con otros métodos (3.7\%), mientras que $19.6 \%$ no pensó aún en cuidarse con algún método anticonceptivo.

Solo el $20.3 \%$ de madres precisaba tener la disposición de utilizar el método anticonceptivo pensado después de su parto, mientras que el $79.7 \%$ no sabía cuándo iniciar su planificación familiar con algún método anticonceptivo. Figura 3.

Hallándose un OR (IC 95 NC) igual a 0.98 al relacionar la información recibida con el uso de algún método de planificación familiar.

Con relación a si habían visto paneles, afiches o murales publicados en el establecimiento de salud, $39.9 \%$ refirió haberlos visto y $60.1 \%$ no los vio. $2.6 \%$ manifestó no haberlos entendido y de las que no los leyeron el $20.7 \%$ manifestó que no le interesaba, $5.5 \%$ no los leyó porque estaban apuradas. Solo un $16.6 \%$ manifestó haber entendido el mensaje de las publicaciones. Tabla 1. 


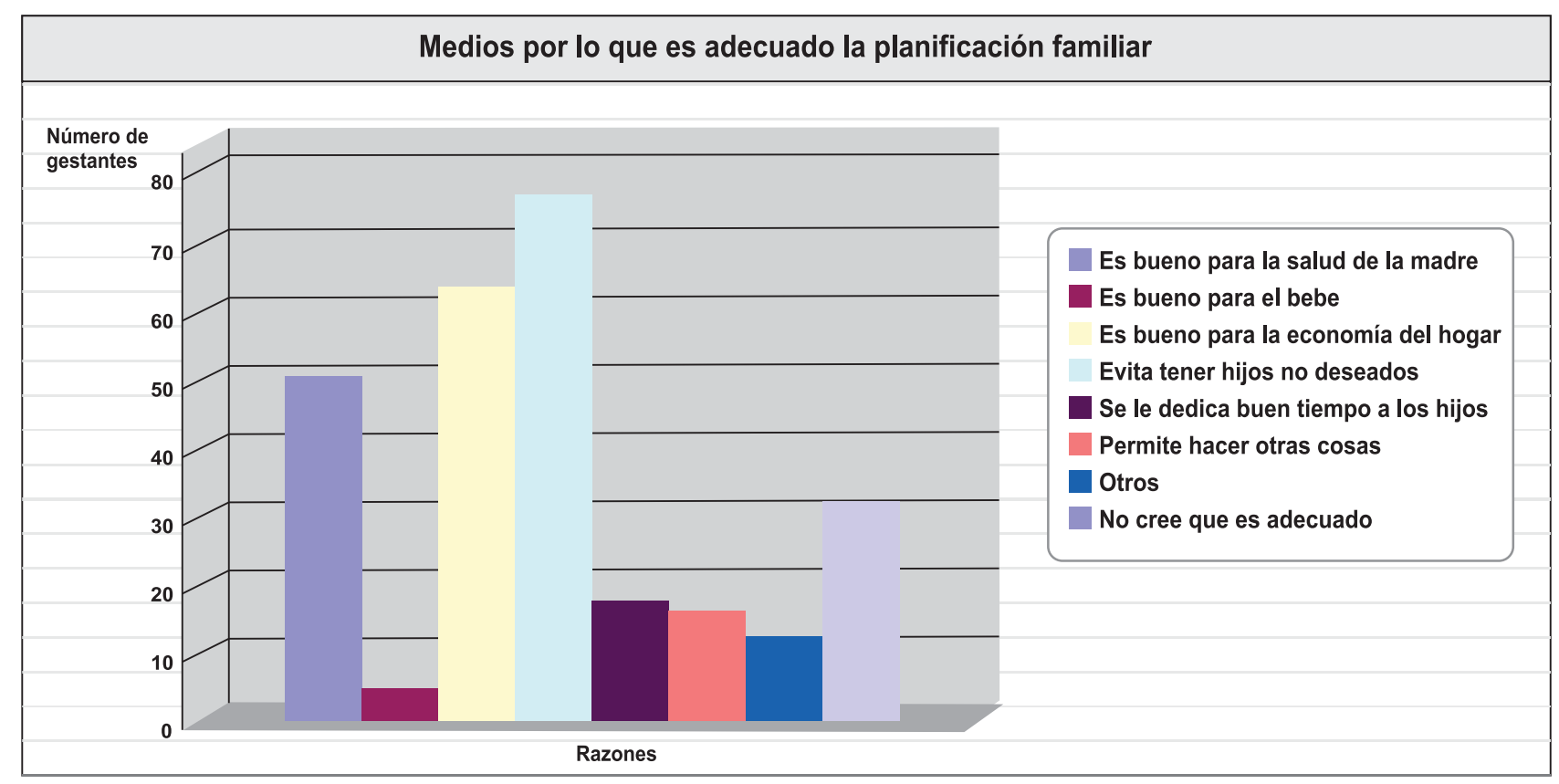

Figura 2. Razones por lo que es adecuado la planificación familiar

\section{Razones por las cuales es necesario los consejos en el embarazo}

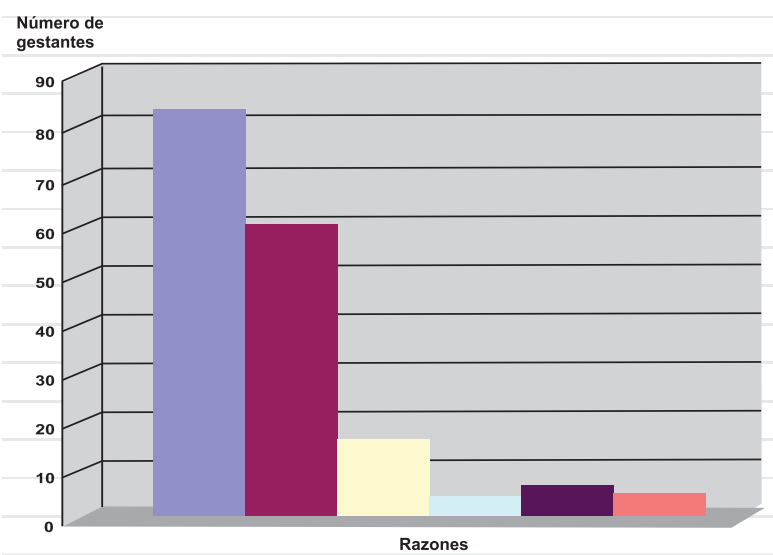

Figura 3. Razones por las cuales es necesario los consejos en el embarazo.

\section{DISCUSIÓN}

El Sector Salud tiene como uno de sus objetivos internalizar en la población de mujeres en edad reproductiva, conductas adecuadas para lograr una maternidad deseada y saludable $(3,4)$.

Desde los $80 \mathrm{~s}$, se puso particular atención a la planificación familiar como una de las intervenciones claves de reducción de riesgos.

Su aplicación se produjo en un contexto en donde el acceso de los métodos anticonceptivos era muy limitado, su acceso en zonas rurales era casi inexistente, la tasa de fecundidad real duplicaba a la tasa de fecundidad deseada en el nivel nacional y prácticamente la triplicaba en las zonas rurales. Se aprobó, por primera vez, la Política Nacional de Población, decretándose la gratuidad de los métodos anticonceptivos e incluyendo la Anticoncepción Quirúrgica Voluntaria, como método anticonceptivo (1).

Sin embargo, observamos que desde el 2000, ha disminuido el uso de los métodos anticonceptivos modernos, aumentando el uso de métodos tradicionales, como la abstinencia periódica $(5,6)$. 
Tabla 1. Percepción de la gestante encuestada sobre PF en el post parto en el embarazo

\begin{tabular}{|c|c|c|c|c|}
\hline Ha pensado cuidarse con algún método anticonceptivo después del parto: & 218 & 80.4 & 53 & 19.6 \\
\hline Hormonal inyectable & 53 & 19.5 & 218 & 80.5 \\
\hline DIU & 24 & 8.9 & 247 & 91.1 \\
\hline Preservativos & 6 & 2.2 & 265 & 97.8 \\
\hline Ha visto paneles o afiches & 178 & 65.7 & 93 & 34.3 \\
\hline - Los ha leído & 108 & 39.8 & 163 & 60.2 \\
\hline - Los ha leído y entendido & 70 & 25.8 & 201 & 74.2 \\
\hline
\end{tabular}

En diversos medios de comunicación (radio, televisión, material escrito: afiches, folletería diversa) se difunden mensajes alusivos a la "maternidad segura", la importancia del "control prenatal", "vacunación" y "planificación familiar" pero no se mide su impacto.

La presente investigación, pretende contribuir con el análisis de costo/ efectividad antes de realizar procesos de intervención que apunten a la difusión de información y, sobre todo, que impliquen gastos elevados para el sector salud (7).

La elección de la Red SJ M, fue tomando en cuenta que congrega una población significativa con alto porcentaje de migración, lo que se pudo apreciar en los resultados de este estudio.

Lo que implica el traslado de patrones culturales y estilos de vida. Esto, explicaría al menos en parte la serie de creencias y prácticas que experimentan $(8,9)$.

A la luz de los resultados, la gran mayoría (74.2\%) sí refirió haber escuchado del tema a través de algún medio de comunicación; sin embargo, a pesar de este alto porcentaje, menos de la cuarta parte de ellas lo percibieron en algún establecimiento de salud y solo alrededor del $13 \%$ manifestó alguna vez haberlo visto o escuchado en algún medio de comunicación masiva, como radio y/o televisión, tanto en señal abierta como cerrada.

Esto llama la atención puesto que es conocido que el precio del diseño y difusión de mensajes publicitarios en estos medios es sumamente alto y que demanda un presupuesto especial, lo cual refleja que los grandes gastos que se han hecho en ellos han sido insuficientes ya que no solo no han tenido impacto sino que probablemente no responden a un buen diseño tanto de las campañas como de los contenidos de los mensajes $(10,11)$.

A pesar de ello, poco más de la tercera parte de madres que, por cualquier medio, escuchó o vio alguna información incluyendo familiares, amigos, colegios y demás, lo asociaban a saber como usar los métodos o cuántos hij os tener.

Esto concuerda con el resultado encontrado al preguntarles sobre por qué consideraban adecuado planificar la familia, poco más de la cuarta parte de ellas solo lo relacionaba con el hecho de evitar tener hijos no deseados, mientras que el resto lo relacionó con otros aspectos no derivados de los mensajes difundidos sino con criterios personales o provenientes de otras fuentes de donde recibieron la información incluyendo familiares, amigos entre otros.

Al investigar sobre el impacto y sostenibilidad del los mensaj es percibidos por las madres que alguna vez los vieron o escucharon, casi dos terceras partes de ellas lo consideró importante, casi igual número manifestó comprenderlo, de ellas a la mayoría le gustó y solo la tercera parte manifestó seguirlos viendo o escuchando. Sin embargo, a pesar de ello y recordando que poco más de la cuarta parte de la población encuestada nunca percibió estos 
mensajes, vemos que esto no guarda relación con sus actitudes y prácticas, debido a que se encontró que solo una pequeña minoría refirió haberse preparado antes de buscar la gestación. Es decir, no existe cultura de prevención (12-14).

Una mayoría de madres, manifestaron su predisposición a cuidarse con algún método anticonceptivo después del parto, como el hormonal en inyectable $(17,18)$, que fue el más mencionado. Sin embargo, al relacionar la información recibida con el uso de algún método de PF, hallamos un OR (IC95 NC) de 0.98; lo que nos demuestra que la comunicación no tuvo impacto sobre las actitudes de las gestantes encuestadas. La mayoría, no tuvo claro cuanto tiempo después del parto debía iniciar la anticoncepción, lo que estaría asociado a no usar método alguno.

Finalmente, se observó que un gran número de madres no notaron la presencia de la publicación de afiches o información en murales o paneles en los mismos establecimientos donde se realizaron las encuestas, casi las dos terceras partes de ellas no los vieron y del grupo restante solo la mitad entendió el contenido y las demás no le prestaron atención por falta de tiempo o por falta de interés, lo que significaría que esta manera de promocionar la salud no sería la más apropiada para lograr en principio llamar la atención de las usuarias del servicio de salud materna y por otro lado para generar el adecuado conocimiento e intención de internalizar conceptos saludables $(15,16)$.

Se debe mantener contacto con la realidad para tener éxito, y la realidad que en verdad cuenta es la que ya existe en la mente del cliente prospecto (17).

En conclusión, el porcentaje de gestantes que recibió información a través de cualquier medio de comunicación sobre la planificación familiar fue muy bajo. Su conocimiento no se relacionó con el contenido de las campañas de promoción alusivas al tema. La fuente de información más efectiva, fue la recibida directamente, sea a través de profesionales de la salud, familiares o amigos.

\section{Fuente de Financiamiento}

El presente trabajo fue autofinanciado por el autor.

\section{Conflictos de interés}

El autor declara no tener ningún conflicto de interés.

\section{REFERENCIAS BIBLIOGRÁFICAS}

1. Plan Estratégico Nacional para la reducción de la mortalidad materna y perinatal 2009-2015. Dirección General de Salud de las Personas, Ministerio de Salud; Ed. Artprint; Lima, Perú. 2009.

2. Ministerio de Salud. Lineamientos de política sectorial para el período 2002-2012 y principios fundamentales para el plan estratégico sectorial del quinquenio agosto 2001 - julio 2006. Primera edición. Lima, Perú. MINSA 2002.

3. Piña F, La Torre L, Alva R.; Funciones obstétricas y neonatales en los establecimientos de salud. Rev Per Obst Enf 2008; 4(1):45-55.

4. Del Bosque S., González J., Del Bosque A., Edits. ¡Manos a la Salud! Mercadotecnia, Comunicación y Publicidad: Herramienta para la Promoción de la Salud. CIESS/OPS México 1998.

5. Ollila E, Koivusalo M, Hemminki E. International actors and population policies in India, with special reference to contraceptive policies. Int J Health Serv 2000; 30: 87-110.

6. Casique I. Empoderamiento femenino y uso de anticonceptivos en México. Boletín Género y Salud en Cifras 2003; 1:13-18.

7. Castro, G. Madres en peligro de muerte. Rev Mensual de Análisis y Propuestas 2008; 7, 22.

8. Salinas J. Health Promotion in Chile: an evaluation of a national plan implementation. Promotion \& Education 2000; 4:13 - 16.

9. Kotler P. Dirección de mercadotecnia: análisis, planteamiento, implementación y control. 8vo. Ed. Prentice-Hall Hispanoamericana S.A. 1995.

10. MacFadyen L, Stead M, Hastings G. A synopsis by the Centre for Social Marketing. Social Marketing, 1999.

11. Ministerio de Salud, Lineamientos de Política Sectorial para el Periodo 2002 - 2012 y Principios Fundamentales para el Plan Estratégico Sectorial del Quinquenio Agosto 2001 - julio 2006. Ministerio de Salud - Dirección general de comunicaciones. Lima, 2002. 
12. Maine, D.; Akalin, M.; Ward, V.; Kamara, A.; Diseño y evaluación de programas para mortalidad materna. Centro para la población y salud familiar. Nueva York: Facultad de Salud Pública. Universidad de Columbia; 1997.

13. Ministerio de Salud. Proyecto 2000, Calidad del servicio de salud desde la percepción de la usuaria. Dirección general de Salud de las personas. Perú. 2000.

14. Ayaga A. Spousal communication and family planning behavior in Navrongo: A longitudinal assessment. Stud Fam Plann 2002; 33: 185-194.

15. Ikechebelu J, Joe-Ikechebelu N, Obiajulu F. Knowledge, attitude and practice of family planning among lgbo women of southeastern Nigeria. J Obstet Gynaecol 2005; 25:792-795.

16. Organización Panamericana de Salud. Política de la Organización Panamericana de la Salud en materia de igualdad de género. Rev Panam Salud Publica 2006; 19:137-140.

17. Ries A, Trout J. Posicionamiento: la batalla por su mente. 1ra. Ed. Mexico. McGraw-Hill Interamericana. 2003.

\section{Correspondencia:}

Sabrina Morales Alvarado

Dirección: Av. Javier Prado Oeste 555 Dpto. 1501 Lima 27, Perú.

Teléfax: +(51 1) 421-0979 / 999-096 282

Correo electrónico: sabrynes@gmail.com

Recibido: 03 de Diciembre de 2013

Aprobado: 20 de Marzo de 2014 